\section{High-Value Cancer Care and the Problem With Surrogate Endpoints in the Quality/Cost Equation}

\author{
Fumiko Chino, MD
}

$\mathbf{V}^{a}$ alue in healthcare is defined as quality divided by cost. Value-based cancer care is the noble ideal that the correct cancer treatment can be recommended for each patient based on careful evaluation of treatment outcomes, patient experience, and cost. ASCO first formed the Task Force on the Cost of Cancer Care in 2007 with the laudable goal of making recommendations for how to balance highquality cancer care with the rising costs of treatment. From this task force, the ASCO Value Framework was launched as a physician-guided tool to facilitate open dialogue between physicians and patients in selecting high-value cancer treatment. ${ }^{1}$

Shared decision-making is crucial in the modern era of cancer therapy, where proliferating options for disease management face off against ballooning treatment costs and increasing patient financial toxicity. Two versions of the ASCO Value Framework calculate net health benefit compared with cost for either advanced disease or potentially curative (adjuvant) clinical scenarios. Both incorporate clinical benefit and toxicity, with potential bonus points given for durable benefit ("tail of the curve") or quality of life improvements, among other clinical scenarios. Costs are reported as both drug acquisition costs and patient payment obligations to honor the financial burden on both health system and individual frameworks.

In this issue of JNCCN, Cheng et $\mathrm{al}^{2}$ analyze the incorporation of surrogate endpoints into the ASCO Value Framework clinical benefit score (CBS). This analysis is timely because surrogate endpoints are increasingly being used for both FDA approval ${ }^{3}$ and in marketing new drugs-which are costly and may not have proven survival benefit-to physicians and patients. One evaluation of FDA-approved drugs based on the surrogate endpoint of response rate (RR) found that the benefit was quite small ( $<40 \%$ for about half of the drugs), and many drugs were granted accelerated approval based on single-arm studies with small sample sizes. ${ }^{4}$ The ASCO Value Framework was designed to increase use of high-value care and foster discussion between physicians and patients; overestimated benefit based on surrogate endpoints may create false expectations and increase financial or physical toxicity without commensurate meaningful clinical gains.

Surrogate endpoints are used to accelerate drug development because differences in overall survival (OS) often take longer to prove, which increases clinical trial costs and duration. ${ }^{5}$ Previous research has shown that most surrogates in oncology had low or modest correlation with OS, suggesting that they should be used with caution when making treatment conclusions. ${ }^{6}$ Despite this, surrogate endpoints are increasingly being used as the primary endpoint for oncology randomized controlled trials, ${ }^{7}$ with growing concern from a contingent of oncologists that we've "put the cart before the horse" and may be placing patients at risk for little to no survival benefit.

Cheng et $\mathrm{al}^{2}$ evaluated the Value Framework CBS of 127 randomized controlled trials in advanced cancer, including 116 that report a hazard ratio (HR) for OS (HR OS) - the current "reference standard." CBS is calculated via the ASCO

See page 1489 for related article.

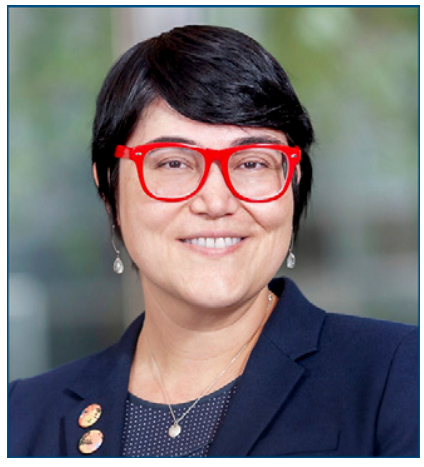

FUMIKO CHINO, MD

Fumiko Chino, MD, is a health services cancer researcher and Assistant Attending Physician in radiation oncology at Memorial Sloan Kettering in New York. Her research is focused on the financial toxicity of cancer care, cancer treatment affordability and value, and shared decision-making. She is passionate about optimizing cancer treatment processes and costs to improve the overall patient experience and decrease disparities in high-quality, high-value care. Dr. Chino tweets at @fumikochino.

doi: $10.6004 /$ jnccn.2019.7372

The ideas and viewpoints expressed in this commentary are those of the author and do not necessarily represent any policy, position, or program of NCCN 
Value Framework equation, with HR OS (or percentage difference in median OS [mOS]) weighted a relative 100\%, progression-free survival (PFS) endpoints weighted $80 \%$, and RR endpoints weighted $70 \%$. The authors found that surrogate-derived CBS overestimate clinical benefit $58 \%$, $68 \%, 77 \%$, and $55 \%$ of the time based on mOS, HR PFS, median PFS (mPFS), and RR, respectively, when compared with CBS calculated from HR OS. The greatest mean absolute error was from mPFS (40.40), far above the previously determined clinically meaningful difference in CBS of $>20$ points. On further analysis, Cheng et $\mathrm{al}^{2}$ found that all surrogates have poor agreement with very wide limits of agreement (LOA) on Bland-Altman plot, with mPFS again having the widest LOA ( -66.31 to 125.93$)$. Unsurprisingly, strong Spearman's rank correlation (0.80) was found between HR OS- and mOSderived CBS, but scores derived from HR PFS, MPFS, and $R R$ were weakly correlated, at 0.38 (statistically significant), $0.20(P=.05)$, and 0.01 (not significant).

Cheng et al ${ }^{2}$ highlight several challenges in using the ASCO Value Framework. First, their analysis shows that using surrogate endpoints as weighted do not allow for accurate assessment of clinical benefit. Unfortunately, given the poor correlation and significant variation in overestimation and underestimation in surrogate-derived CBS, a simple correction (eg, reweighting endpoints) does not improve validity. In real-world practice, this becomes even more complex, because randomized clinical trials for 2 treatments may have reported 2 different surrogate endpoints, making comparison of calculated CBS even more disparate.

After its introduction in 2015, the ASCO Value Framework received substantial feedback and was revised in 2016 with changes to how clinical benefit, toxicity, and costs were reported, including making HR OS the preferred endpoint for CBS calculation. ${ }^{1}$ The ASCO Task Force addressed critiques regarding possible obstacles to use in daily practice, including practicality and patient personalization, and laid out plans to develop a user-friendly software application.
In addition to the current study by Cheng et al, ${ }^{2}$ previously published evaluations of the ASCO Value Framework include concern that the framework does not consistently provide an absolute measure of survival benefit. This lack makes it difficult to incorporate into a cost-effectiveness framework that is primarily assessed via incremental quality-adjusted life-years. ${ }^{8}$ Additional concerns include lack of clinically relevant validated thresholds for net health benefit (NHB) scores, making differences in these scores difficult to interpret into what is clinically meaningful for patients. ${ }^{9}$ In addition, NHB scores calculated from placebo-controlled trials are much higher than those calculated from trials randomized between 2 active treatments, again making comparisons impossible. Even the utility of using HR OS as the reference standard is not without flaws, especially in the context of advanced disease in which analysis of OS is increasingly confounded by the effect of salvage therapies used after disease progression. ${ }^{10}$

Ultimately, no value framework can accurately incorporate all elements that are important in the value equation. The idea of "quality" includes many concepts, such as access, healthcare equity, and patient-reported outcomes, which may be hard to translate into a concrete numerical score. "Value" itself is a nebulous concept that entirely depends on which stakeholder (patient, provider, insurer, policymaker) is setting the agenda.

Regardless of the fundamental shortcuts inherent in making a generalizable value framework, we must acknowledge that the use of surrogate endpoints for accessing clinical benefit will continue. Cheng et al's ${ }^{2}$ work presents the flaws and potential bias inherent in their use and helps inform interpretation of results; careful analyses such as these help move the discussion forward and may lead to incremental improvements in the ASCO Value Framework. Ultimately, incorporation of surrogate endpoints as components of value are a pragmatic sacrifice. We use them because we often have no other options. A flawed system for evaluating value is still better than no system at all.

\section{References}

1. Schnipper LE, Davidson NE, Wollins DS, et al. Updating the American Society of Clinical Oncology Value Framework: revisions and reflections in response to comments received. J Clin Oncol 2016;34: 2925-2934.

2. Cheng $S$, Cheung MC, Jiang DM, et al. Are surrogate endpoints unbiased metrics in clinical benefit scores of the ASCO Value Framework? J Natl Compr Canc Netw 2019;17:1489-1496.

3. Kim C, Prasad V. Cancer drugs approved on the basis of a surrogate end point and subsequent overall survival: an analysis of 5 years of US Food and Drug Administration approvals. JAMA Intern Med 2015;175:1992-1994.

4. Chen EY, Raghunathan V, Prasad V. An overview of cancer drugs approved by the US Food and Drug Administration based on the surrogate end point of response rate. JAMA Intern Med 2019;179:915-921.

5. Chen EY, Joshi SK, Tran A, Prasad V. Estimation of study time reduction using surrogate end points rather than overall survival in oncology clinical trials. JAMA Intern Med 2019;179:642-647.

6. Haslam A, Hey SP, Gill J, Prasad V. A systematic review of trial-level meta-analyses measuring the strength of association between surrogate

end-points and overall survival in oncology. Eur J Cancer 2019;106: 196-211.

7. Kay A, Higgins J, Day AG, et al. Randomized controlled trials in the era of molecular oncology: methodology, biomarkers, and end points. Ann Oncol 2012;23:1646-1651.

8. Saluja R, Everest L, Cheng S, et al. Assessment of whether the American Society of Clinical Oncology's Value Framework and the European Society for Medical Oncology's Magnitude of Clinical Benefit Scale measure absolute or relative clinical survival benefit: a meta-analysis of randomized clinical trials [published online May 16, 2019]. JAMA Oncol. doi: 10.1001/ jamaoncol.2019.0818

9. Seymour EK, Schiffer CA, de Souza JA. Challenges in the clinical application of the American Society of Clinical Oncology Value Framework: a Medicare cost-benefit analysis in chronic lymphocytic leukemia. J Oncol Pract 2017;13:e1002-1011.

10. Saad ED, Buyse M. Statistical controversies in clinical research: end points other than overall survival are vital for regulatory approval of anticancer agents. Ann Oncol 2016;27:373-378. 